\title{
Synthesis and identification of a metal-organic framework of zirconium and a carboxylate ligand in DMF
}

\author{
Mahdiyeh Jamshidi Eyni, Faranak Manteghi* \\ Department of Chemistry, Iran University of Science \& Technology, Tehran, Iran
}

\section{Abstract}

Metal-organic frameworks are a new class of advanced porous materials, which have several important characteristics, such as highly periodical, diverse and designable structures, high porosity, unique and modifiable pore surface, as well as framework flexibility. Metal-organic frameworks (MOFs) are composed of organic linkers and inorganic nodes. For the past decade, they have been extensively studied because of their extraordinary porosity that makes them suitable for catalysis, gas separation, sensors, biomedical science, and supercapacitors. In particular, robust MOFs with strong coordination covalent bonds, such as those between metal atoms and carboxylates, are of interest. In this context, Zr-based MOFs, such as UiO-66 (nominal composition: $\mathrm{Zr}_{6} \mathrm{O}_{4}(\mathrm{OH})_{4}(\mathrm{BDC})_{6}$; $\mathrm{BDC}=$ benzene-1,4dicarboxylate) featured zirconium oxide cluster $\left(\mathrm{Zr}_{6} \mathrm{O}_{8}\right)$ secondary building unit (SBU) are known for their high mechanical, chemical, and thermal stabilities.

In this research, $\mathrm{ZrCl}_{4}$ and (benzene-1,4-dicarboxylate) BDC as linker were reacted in solvent (Dimethylformamide) by mechanochemical method. The characterization of the obtained MOF was conducted by XRD, FTIR and SEM.

Keywords: Metal-organic framework, Zirconium, Linker, BDC.

\section{Introduction}


Metal-organic frameworks or MOFs, have emerged as an extensive class of crystalline materials with ultrahigh porosity (up to $90 \%$ free volume) and enormous internal surface areas, extending beyond $6000 \mathrm{~m}^{2} / \mathrm{g}$. These properties, together with the extraordinary degree of variability for both the organic and inorganic components of their structures, make MOFs of interest for potential applications in clean energy, most significantly as storage media for gases such as hydrogen and methane, and as highcapacity adsorbents to meet various separation needs. Additional applications in membranes, thin film devices, catalysis, and biomedical imaging are increasingly gaining importance. On a fundamental level, MOFs epitomize the beauty of chemical structures and the power of combining organic and inorganic chemistry, two disciplines often regarded as disparate. Since the 1990s, this area of chemistry has experienced an almost unparalleled growth, as evidenced by not only the sheer number of research papers published but also the ever-expanding scope of the research [1].

Among the large family of metal-organic frameworks (MOFs), Zr-based MOFs, which exhibit rich structure types, outstanding stability, intriguing properties and functions, are foreseen as one of the most promising MOF materials for practical applications. Although this specific type of MOF is still in its early stage of development, significant progress has been made in recent years. Herein, advances in Zr-MOFs since 2008 are summarized and reviewed from three aspects: design and synthesis, structure, and applications. Four synthesis strategies implemented in building and/or modifying Zr-MOFs as well as their scale-up preparation under green and industrially feasible conditions are illustrated first. Zr-MOFs with various structural types are then classified and discussed in terms of different $\mathrm{Zr}$ based secondary building units and organic ligands. Finally, applications of $\mathrm{Zr}-\mathrm{MOF}$ in catalysis, molecule adsorption and separation, drug delivery, and fluorescence sensing, and as porous carriers are highlighted [2]. The solvent is one of the most important parameters in the synthesis of MOFs, which has been investigated to a lesser extent in zeolite synthesis. Although most often no direct interaction with the framework is observed, solvents are almost always incorporated in the as-synthesized MOF structures and thus act as space-filling molecules. Structure- 
directing agents (SDAs) have been mostly used in the field of zeolite synthesis, where they act as counterions for charge balance, as spacefilling molecules, or as "true" templates, in the case of well-defined host_guest interactions. SDAs have also been employed for the preparation of porous MOFs, but it is often hard to identify the role of the solvent as pore-filling molecule (SDA) or as pure reaction medium [3].

\section{Experimental}

\section{Materials}

In this study, $\mathrm{ZrCl}_{4}$ and $\mathrm{BDC}$ (benzene-1,4-dicarboxylate, 98.9\%) was supplied by Sigma-Aldrich and DMF (dimethylformamide, 99\%) and chloroform, 99\%) by Merck.

\section{Preparation of UiO-66}

$\mathrm{ZrCl}_{4}$ as a metallic source and terephthalic acid as a ligand were mixed well in a mortar. The resulting mixture was then transferred into a ball mill, first ground at $15 \mathrm{~Hz}$ for $10 \mathrm{~min}$ for complete mixing and reacting, and then 3 drops of dimethylformamide were added to accelerate the reaction and create conditions for better nucleation. In the next step, the milling process continued for three times, at least $15 \mathrm{~min}$ at $15 \mathrm{~Hz}$, then the mixture together with dimethylformamide was washed to remove impurities. Afterwards, the powder was washed with chloroform and finally dried in a vacuum oven.

\section{Results and discussion}

FT-IR spectrum of UiO-66

As shown in Fig. 1, carboxylic acid has a broad $\mathrm{OH}$ peak at 2800-3400 $\mathrm{cm}^{-1}$ and a C-O band at $1550-1600 \mathrm{~cm}^{-1}, 1250-1350 \mathrm{~cm}^{-1}$ and $\mathrm{O}-\mathrm{H}$ band at $910-950 \mathrm{~cm}^{-1}$. 


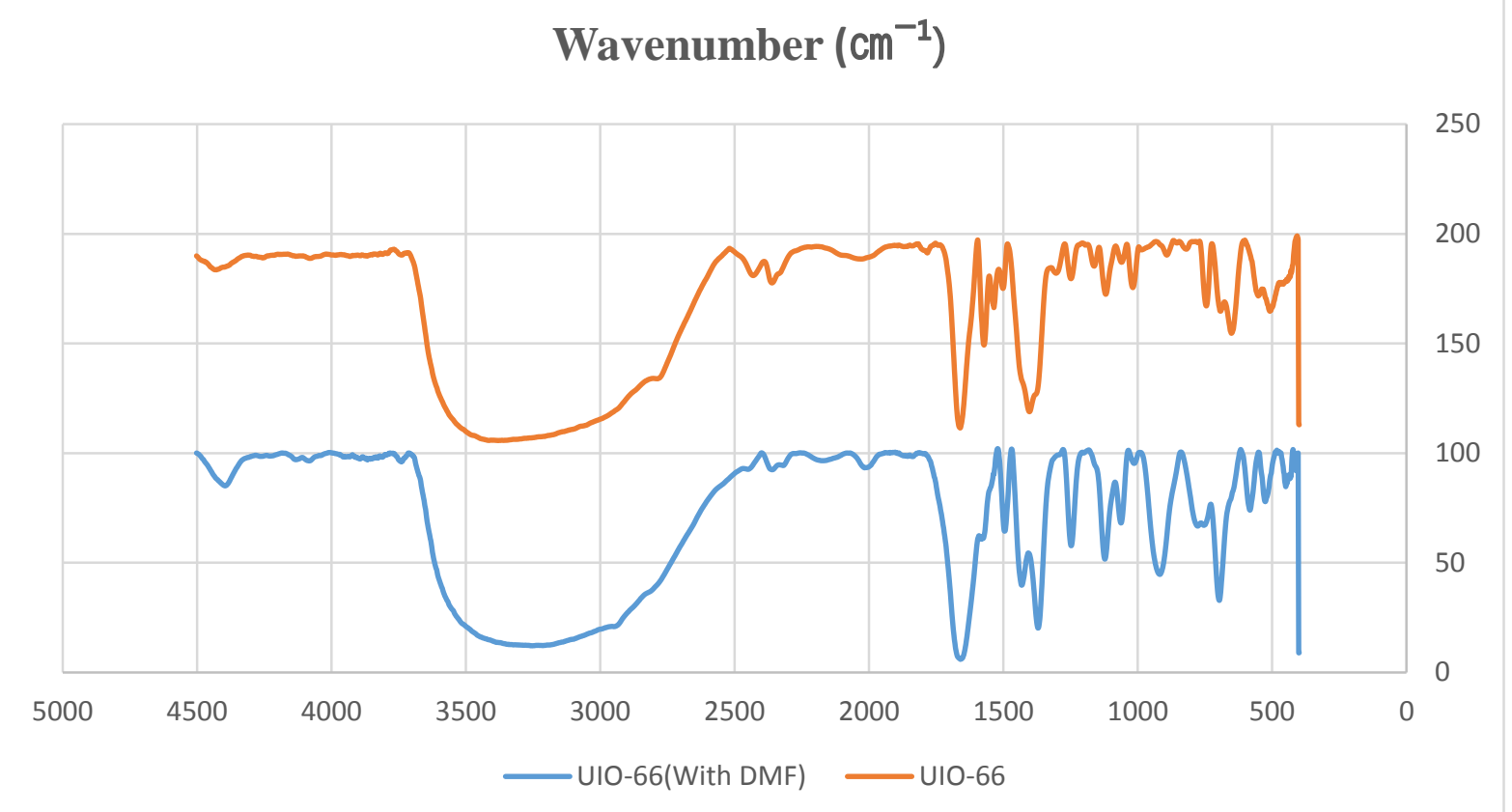

Fig. 1: FT-IR spectrum of UiO-66

\section{XRD Pattern of UiO-66}

In Fig. 2, X-ray diffraction pattern reveals hexagonal structure.

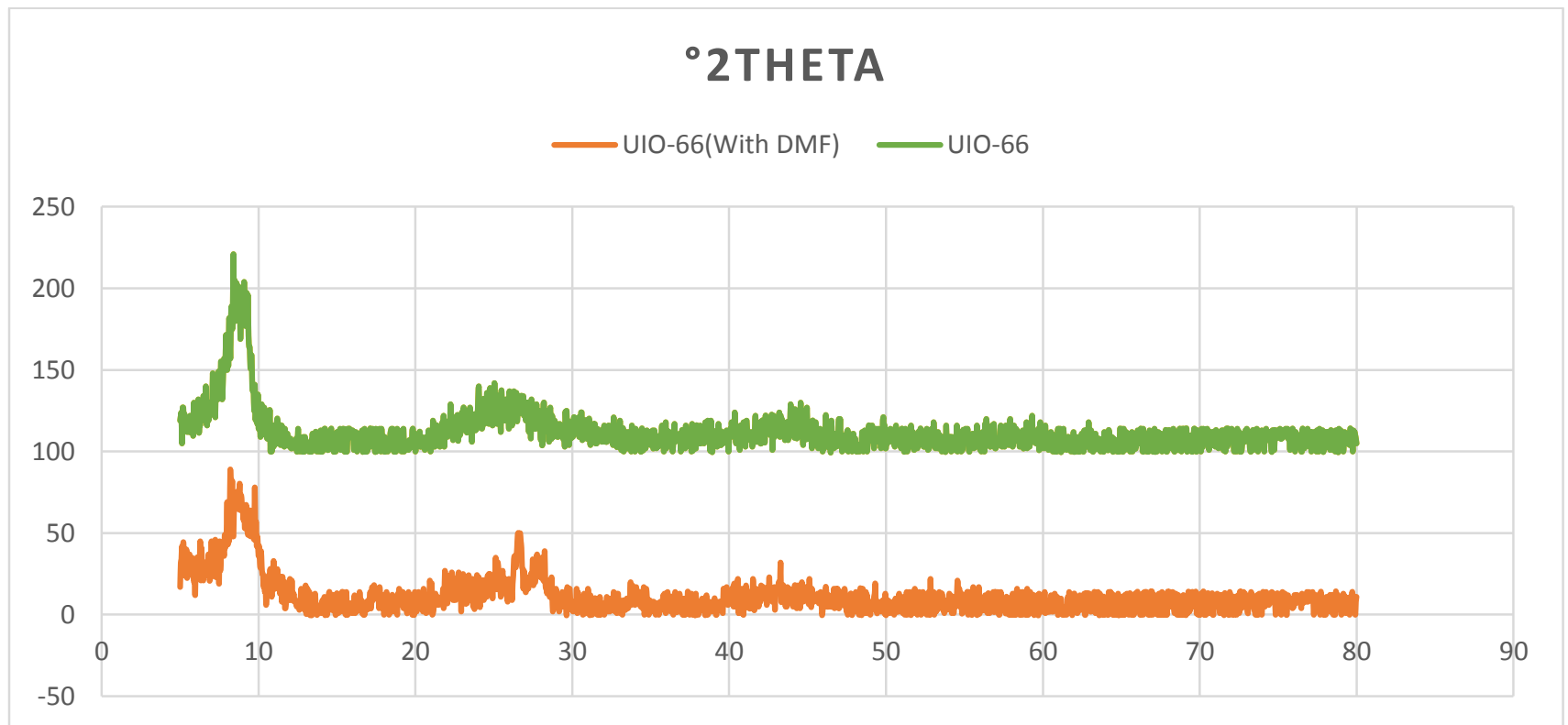

Fig. 2: XRD pattern of UiO-66. 
SEM image of UiO-66

The morphologies of the title MOF were characterized by scanning electron microscopy. As shown in Fig. 3, the particles size are in the range of nanometers.

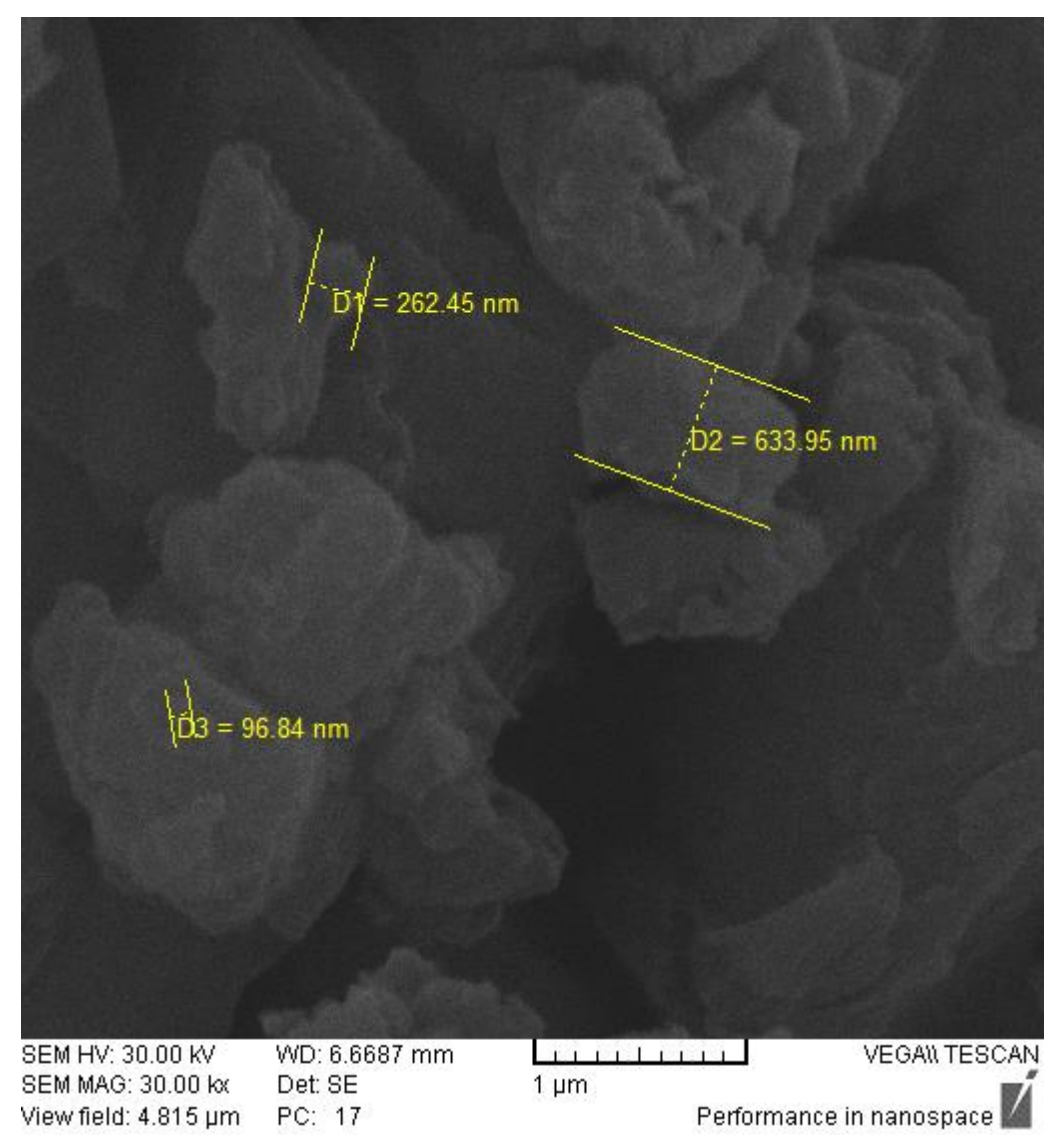

Fig. 3: SEM image of UiO-66.

\section{Conclusions}

In summary, UiO-66 was synthesized mechanochemically. Using dimethylformamide, makes better nucleation and more crystalline particles, and increases the cavities in the structure of the metalorganic framework. 


\section{References}

[1] Stock Norbert, Biswas Shyam. (2012). Synthesis of Metal-Organic Frameworks (MOFs):

Routes to Various MOF Topologies, Morphologies, and Composites. Chemistry Reviews, 112 (2), 933-969.

[2] Yan Bai, Yibo Dou, Lin-Hua Xie, William Rutledge, Jian-Rong Li and Hong-Cai Zhoub. (2016). Zr-based metal-organic frameworks: design, synthesis, structure, and applications, The Journal Chemical Society Reviews, 45, 2327-2367.

[3] Ling Sanliang, Slater Ben. (2015). Unusually Large Band Gap Changes in Breathing MetalOrganic Framework Materials. The Journal of Physical Chemistry C, 119 (29), 16667-16677. 